\title{
Identification of materials with strong magnetostructural coupling using computational high-throughput screening
}

\author{
Luis Casillas-Trujillo $\odot$, Rickard Armiento $\odot$, and Björn Alling $\odot$ \\ Department of Physics, Chemistry and Biology (IFM), Linköping University, 58183 Linköping, Sweden
}

(Received 21 January 2021; accepted 25 February 2021; published 23 March 2021)

\begin{abstract}
Important phenomena such as magnetostriction, magnetocaloric, and magnetoelectric effects arise from, or could be enhanced by, the coupling of magnetic and structural degrees of freedom. The coupling of spin and lattice also influence transport and structural properties in magnetic materials, in particular around phase transitions. In this paper we propose a method for screening materials for a strong magnetostructural coupling by assessing the effect of the local magnetic configuration on the atomic forces using density functional theory. We have employed the disordered local moment approach in a supercell formulation to probe different magnetic local configurations and their forces and performed a high-throughput search on binary and ternary compounds available in the Crystallography Open Database. We identify a list of materials with a strong spin-lattice coupling out of which several are already known to display magnetolattice coupling phenomena such as $\mathrm{Fe}_{3} \mathrm{O}_{4}$ and CrN. Others, such as $\mathrm{Mn}_{2} \mathrm{CrO}_{4}$ and $\mathrm{CaFe}_{7} \mathrm{O}_{11}$, have been less studied and have yet to reveal their potentials in experiments and applications.
\end{abstract}

DOI: 10.1103/PhysRevMaterials.5.034417

\section{INTRODUCTION}

Magnetic materials are used in a wide range of applications including novel and attractive technologies where material improvements are desired. Yet, they also represent a great challenge in fundamental physics and computational simulations slowing down the phase of development in areas such as magnetic refrigeration, permanent magnets, and steels. In particular, in theoretical first-principles research these issues manifest themselves in materials-specific computational and numerical challenges, which is reflected in the relatively low number of theoretical high-throughput magnetic material studies. Even standard low-throughput magnetic calculations are challenging, often leading to simplifying assumptions, such as neglecting magnetic excitations and disorder and even approximating the paramagnetic state as nonmagnetic [1,2]. When the complexity of magnetism is indeed investigated, lattice degrees of freedom, such as vibrations or lattice relaxations, are often neglected [3]. However, many of the technologically attractive phenomena displayed by magnetic materials originate from the coupling between magnetic, electronic, and structural degrees of freedom. Magnetostriction, the magnetocaloric effect (MCE), and the magnetoelectric effect are already being used in technological applications and play a fundamental role in the development of new technologies [4].

Published by the American Physical Society under the terms of the Creative Commons Attribution 4.0 International license. Further distribution of this work must maintain attribution to the author $(s)$ and the published article's title, journal citation, and DOI. Funded by Bibsam.
The coupling phenomenon between the magnetic field and the strain field of magnetostrictive materials makes them widely used in sensors and actuators [5]; applications range over, e.g., load cells, torque meters, pressure gauges, tensile stress sensors, thin-film thickness sensors, and dynamic stress sensors [6]. Taking advantage of this phenomenon can also improve or revolutionize well-established technologies. Magnetostrictive transducers were recently developed with the unique characteristic that they do not require direct wiring between the magnetostrictive material and the sensing/actuating solenoids [7].

The magnetoelectric effect has applications in memory devices, magnetic field sensors, and spintronics [8-11] by providing efficient control of magnetism by an electric field. Switching the orientation of magnetization using an electric field has a substantially lower energy requirement [12]. The ability to modify the magnetization using a low-magnitude electric field (and vice versa) is of great interest for speeding up the processing rate of memory devices, and also for the design of very sensitive stress sensors [13].

Magnetocaloric materials have a strong coupling between the crystallographic structure and magnetism. A magnetic field can induce a simultaneous change of magnetic and lattice entropies [14]. In the well-known case of $\mathrm{Gd}_{5} \mathrm{Si}_{2} \mathrm{Ge}_{2}$, in the magnetic field induced transformation from the paramagnetic monoclinic phase to the ferromagnetic orthorhombic phase, the entropy change due to structural transition contributes to more than half of the total entropy change [14]. Magnetic refrigeration, based on the MCE, has been proposed as an energy efficient and environmentally friendly alternative to vapor-compression refrigeration [15]. Developments in magnetocaloric materials in recent years have led to the first prototype systems [16], yet material limitations, such as component weight and cost, are still an obstacle for allowing 
large-scale commercial distribution. Besides the application of the MCE to magnetic refrigeration equipment, magnetic heat pumps, and magnetocaloric energy conversion machines, the MCE has shown great promise for medical applications, targeted drug delivery, and the destruction of cancer cells [15].

Besides these phenomena, the coupling of magnetic and structural degrees of freedom impacts material properties such as thermal conductivity. The anomalous thermal conductivity exhibited by magnetic semiconductors has been recently attributed to the shortening of the lifetime of acoustic phonons due to spin-phonon coupling [17]. The thermal conductivity of $\mathrm{CrN}$ displays an extremely different temperature dependence below and above the magnetic transition temperature, remaining almost constant above the transition temperature. The dynamics of the spin system severely impact lattice vibrations, significantly reducing the phonon lifetime of the acoustic modes above the transition temperature. This behavior has also been observed in one of the top candidates found in the high-throughput study, $\mathrm{Fe}_{3} \mathrm{O}_{4}$, which displays a strong change in the behavior of thermal conductivity and thermal diffusivity close to the transition temperature [18]. The relationship between structure and magnetism is also present in crystallographic defects, where the change in environment carries a concomitant change in magnetic moment as in the case for screw dislocation in the paramagnetic state [19].

High-throughput methods have proven to be a powerful tool in materials science, allowing the discovery and design of new materials [20-23]. Furthermore, theoretical high-throughput calculations can efficiently screen a large quantity of materials without experimental synthesis [24], representing savings in time and money by pointing to the most promising candidates that can be further examined by experimental investigations. While high-throughput methods sacrifice accuracy for automated analysis of a large number of compounds, they can produce more candidates than highaccuracy low-throughput studies. The vast amount of material data already available in databases offers the possibility to efficiently screen a large quantity of materials. As such, it enables probing materials for different target properties. Hence, it is possible to find new applications for well-known materials, for example, the case of $\mathrm{UO}_{2}$ which is famously known for its use as nuclear fuel, but is also the hardest piezomagnet known [25].

The paramount importance of magnetic materials has motivated several experimental high-throughput searches to find better performing magnets [24,26] and the development of machine learning approaches to improve high-throughput searches of rare-earth free permanent magnets [27]. As mentioned before, despite all the fascinating properties displayed by magnetic materials, there have been few theoretical high-throughput calculations. Recently Horton et al. [28] performed a high-throughput study to assess the magnetic order of the ground state in inorganic materials, and Bocarsly et al. [29] screened magnetocaloric compounds. In this work we conduct a high-throughput search to find materials with strong magnetostructural coupling using density functional theory (DFT) calculations within the High-Throughput Toolkit (httk) framework [30]. To assess magnetostructural coupling we suggest the following descriptor: the effect that the different local magnetic environment in a disordered magnetic structure has on the force acting on each atom in the structure. This is a different effect compared to the magnetostructural coupling happening through the spin-orbit coupling. The relativistic spin-orbit coupling is weak on an atomic scale but important in deciding the global magnetization direction with respect to the crystal structure in ordered magnetic states, manifested in the magnetic anisotropy. By using here the magnetically disordered state we probe the atomic-scale, electronic origin, of spin-lattice coupling in a model of the paramagnetic state of direct relevance for the magnetocaloric effect and thermal conductivity at high temperature. But simultaneously, the use of the symmetry breaking of the disorder acts as a path to discover spin-lattice coupling, of possible importance also for ordered magnetic states, that might be hidden in calculations if they are performed on highly symmetric ground states.

\section{METHODS}

\section{A. Disordered local moment}

The simulation of the paramagnetic state by first-principles calculations is challenging and, in some cases, has been approximated by the nonmagnetic state. The interatomic bond strengths can differ greatly between magnetic and nonmagnetic states [31], leading to incorrect results [1,2]. In order to properly describe the paramagnetic state, one can employ the disordered local moment approach (DLM) [3] as implemented in a supercell framework $[32,33]$. The real magnetization density in many magnetic materials can be described in terms of magnetic moments localized close to the atoms. The PM case can then be viewed as a disordered distribution of such local moments with no long-range order, and sufficiently above the critical temperature approximated with the fully disordered state also lacking short-range order (SRO), i.e.,

$$
\operatorname{DLM}: \frac{1}{N} \sum_{i, j \in \alpha} \mathbf{e}_{i} \cdot \mathbf{e}_{j}=0, \quad \forall \alpha,
$$

where $N$ is the number of magnetic pairs in the summation; $i$ and $j$ denote the atoms in question belonging to a specific coordination shell $\alpha . \mathbf{e}_{i}$ and $\mathbf{e}_{j}$ are the unit vectors in the direction that maximizes the magnetization density around atom $i$ or $j$, i.e., the direction of the atomic magnetic moment on atom $i$ or $j$.

The DLM result can be obtained with a supercell constructed using the special quasirandom structures (SQS) approach [34] that fulfills Eq. (1) by construction for several closest coordination shells, or by sampling and averaging over a large number of randomly chosen supercell configurations [33]. The latter is more practical in the present case as the SQS approach demands the design of a separate SQS for each of the hundreds of crystal structures studied here. For the high-throughput screening, we have chosen to sample a few random configurations since this represents savings in time and computer resources, and the descriptor is not evaluated with a need for full convergency with respect to magnetic SRO-statistics. The DLM approach allows formulating a consistent electronic structure based thermodynamic theory that accounts for the interplay between the configurational and magnetic degrees of freedom [35]. The DLM approach can 


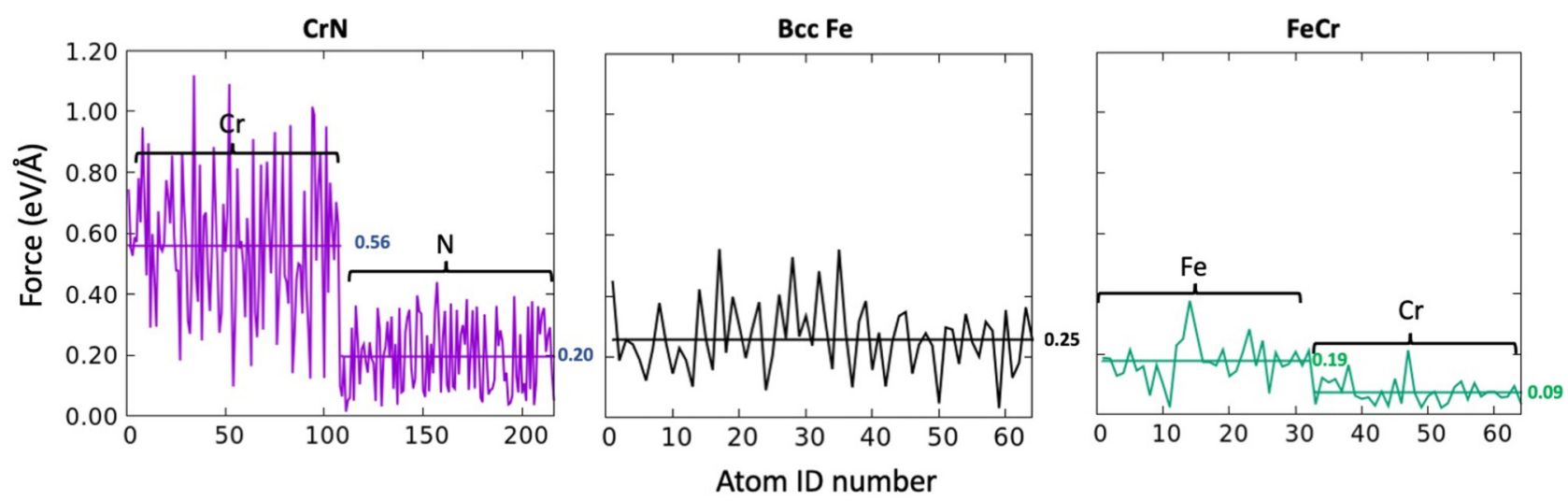

FIG. 1. Comparison of forces in the paramagnetic state between $\mathrm{CrN}$, bcc Fe, and bcc $\mathrm{Fe}_{0.5} \mathrm{Cr}_{0.5}$ alloy.

be employed with either collinear or noncollinear magnetic moments [33]. In general, noncollinear calculations give a smaller magnitude for the forces than collinear calculations [36], yet the choice of approach has no effect on the force based hierarchy among the probed compounds. Noncollinear calculations are significantly more computationally expensive than collinear calculations, and therefore we have chosen to perform the calculations with collinear magnetic moments.

\section{B. Descriptor: Interatomic forces induced by disordered magnetism}

Filipetti and Hill [37] proposed the concept of magnetic stress, defined as the stress associated with the change in spin ordering. This concept establishes a connection between magnetic ordering and structural properties. Bocarsly et al. [38] suggested that magnetostructural coupling has an important role behind the magnetocaloric behavior, and employed the magnetic deformation [29], a DFT based descriptor defined as the degree of lattice deformation between nonmagnetic and ferromagnetic unit cells, to assess the magnetocaloric effect in $\mathrm{MnB}$ and $\mathrm{FeB}$. They found that while $\mathrm{MnB}$ is isostructural to $\mathrm{FeB}$ and has similar magnetic properties, the former possesses a larger magnetic deformation and has a larger magnetocaloric effect, whereas the latter displayed little magnetostructural coupling.

Yet these approaches do not capture the effect of magnetic disorder and local magnetic environment effect. Important structural transitions occur at the critical temperature where the system goes from paramagnetic to some ordered magnetic state, and as such one should investigate the paramagnetic state. Therefore, we use the force induced on each atom by the local magnetic configuration in the paramagnetic state as a descriptor to find materials with strong magnetostructural coupling. We suggest that if the change in the local magnetic configurations produces a large change in the force value, it is an indicator of strong magnetostructural coupling. With this approach we isolate the effect of magnetism on interatomic forces, and the relief of these forces may come as structural transformations or it may manifest itself as a dynamical coupling between lattice and spin dynamics [17]. In principle, this approach can be employed between different ordered magnetic states. However, due to the symmetry in ordered cases, some of the spin-lattice coupling induced forces can be hidden. Employing the disordered magnetic state gives direct insight into the physics around the order-disorder transition, critical in many applications of magnetic materials.

To illustrate the dependence of the force acting on each atom on the magnetic environment we use chromium nitride $(\mathrm{CrN})$, bcc-Fe, and bcc- $\mathrm{Fe}_{0.5} \mathrm{Cr}_{0.5}$. $\mathrm{CrN}$ is a prototype material with strong magnetostructural interactions. At room temperature it has a rocksalt structure and paramagnetic state. Below its Néel temperature $\left(T_{n}=273 \mathrm{~K}\right)$ it undergoes a structural and magnetic transition to antiferromagnetic (AFM) ordering and to an orthorhombic structure with space group Pnma [39-42]. In Fig. 1 we compare and contrast the behavior of $\mathrm{CrN}$, bcc $\mathrm{Fe}$, and bcc $\mathrm{Fe}_{0.5} \mathrm{Cr}_{0.5}$ random alloy, where we plot the magnitude of the net force acting on each atom sitting on ideal lattice positions but with a disordered magnetic configuration. In $\mathrm{CrN}$, it can be seen that there is a large oscillation in the force values of $\mathrm{Cr}$ atoms, indicating a strong dependence on the local magnetic configuration, having a range of $\sim 1 \mathrm{eV} / \AA$ and an average value of $0.56 \mathrm{eV} / \AA$ for the $\mathrm{Cr}$ atoms, while the force on the nonmagnetic $\mathrm{N}$ atoms is considerably lower with an average of $0.20 \mathrm{eV} / \AA$. For bcc $\mathrm{Fe}$ and $\mathrm{FeCr}$, the force on $\mathrm{Fe}$ atoms has a similar value, with $0.25 \mathrm{eV} / \AA$ for bcc $\mathrm{Fe}$ and $0.19 \mathrm{eV} / \AA$ for $\mathrm{FeCr}$, and an average value of $0.09 \mathrm{eV} / \AA$ for $\mathrm{Cr}$ atoms in $\mathrm{FeCr}$. Thus, $\mathrm{Cr}$ atoms in $\mathrm{CrN}$ present a distinctively larger average value and force range. These force values highlight that the descriptor captures the combined effect of the symmetry-broken disordered magnetic state with different magnetic local environments of each atom and the general bond strength of the material which is high for the ceramic CrN. Hence, the descriptor is suitable for searching for materials with strong spin-lattice coupling for use as multiferroic, magnetocaloric, and magnetostrictive materials.

\section{Computational details}

The set of compounds to perform the high-throughput calculations are obtained from the Crystallography Open Database (COD) [43]. We have retrieved binary and ternary compounds that contain the magnetic elements $\mathrm{Ni}, \mathrm{Cr}, \mathrm{Co}$, $\mathrm{Fe}$, and $\mathrm{Mn}$. From this set we select compounds with at least $20 \%$ magnetic elements per formula unit. To have enough atoms for magnetic disorder, we require supercells with at least ten magnetic atoms. Thus, if the original structure is small, a supercell of at least ten magnetic atoms is created by 


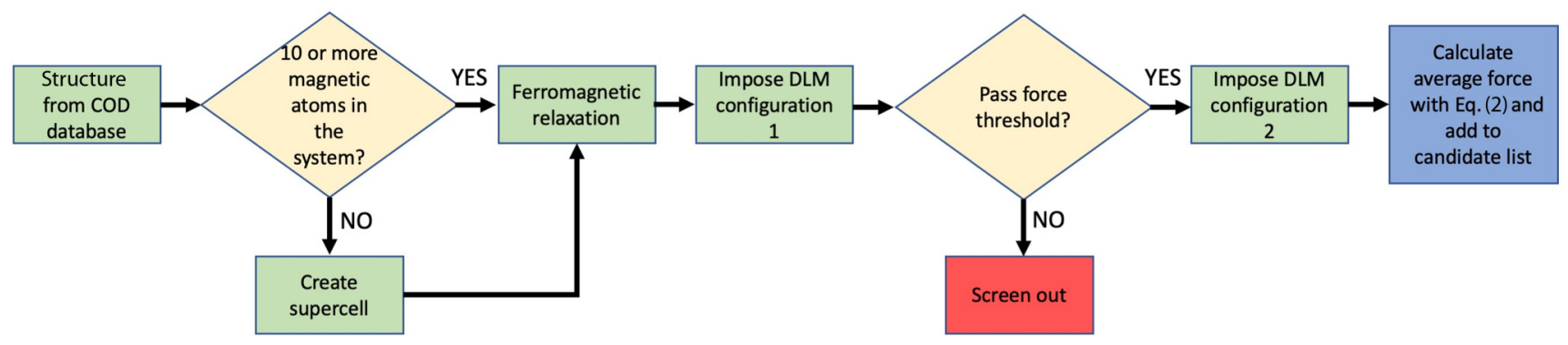

FIG. 2. Schematic representation of the screening process.

multiplying the unit cell in the $x, y$, and $z$ directions until the minimum size is attained. The high-throughput calculations are performed using the $h t t k$ which interfaces with the Vienna $a b$ initio package (VASP) $[44,45]$ to perform the $a b$ initio collinear spin-polarized calculations with the Perdew-BurkeErnzenhof generalized gradient approximation [46] to model the exchange-correlation effects and the projector augmented wave basis [47]. The structure of the selected compounds was first relaxed using a ferromagnetic configuration to assess the structural differences between the structure reported in the database and the relaxed structures from first-principles calculations. The energy cutoff is set to $600 \mathrm{eV}$ for all compounds and we employed a Monkhorst-Pack $k$-point grid with $20 k$ points/A density for all supercells. We do not go beyond standard DFT calculations, and therefore we do not employ $\mathrm{DFT}+U$ corrections and exclude structures with chemical disorder, e.g., atomic sites with partial occupancies.

Once we obtain the ferromagnetically relaxed structure, we impose a randomly assigned DLM magnetic configuration with local magnetic moments initiated with a 3 Bohr magneton $\left(\mu_{\mathrm{B}}\right)$ magnitude. We then perform a static calculation to obtain the forces on the atoms. We have set a threshold of $0.3 \mathrm{eV} / \AA$ to decide if the material displays large forces. This value was set during our preliminary calculations which included 100 compounds chosen by hand. If any of the individual forces in the material passes the threshold, we perform a second calculation with a different DLM configuration, with the goal of comparing the difference in forces caused by different disordered configurations that differ only by local magnetic configurations while having the same lack of longrange order and magnetic symmetry. Otherwise, we deem the material as having weak magnetolattice coupling and it is screened out. This screening procedure is schematically shown in Fig. 2. In these collinear calculations, the sign of the magnetic moments is not constrained. After the calculations, we consider as magnetic species the elements that possess an average magnetic moment above $0.05 \mu_{\mathrm{B}}$. If the final magnetic moment of a considerable fraction of these magnetic atoms changes sign, it may be an indication of a strong magnetic ordering tendency, could reduce the difference in local magnetic environments, and cast some questions if its forces can be compared to the nonflipped, fully disordered cases. Obviously, if $50 \%$ of the moments would flip, any ground state ordered magnetic configuration could have appeared. But for our randomly assigned starting configuration, even with fewer flips, disorder might be drastically reduced. For this reason, we focus here on the materials with fewer than $20 \%$ spin flips. However, all materials that pass the force criteria are presented in the Supplemental Material [48] together with the fraction of spin flips.

\section{RESULTS}

\section{A. Single elements}

First, we investigate the magnetically induced forces for single magnetic elements. We have considered bcc $\mathrm{Cr}$, fcc $\mathrm{Mn}$, bcc Mn, fcc Fe, bcc Fe, hep Co, fcc Co, fcc Ni and, hcp $\mathrm{Gd}$, and perform calculations with unconstrained magnetic moments, magnetic moments constrained in the up/down direction, and with constraints in both direction and magnitude. Henceforth, we will refer to the latter as fully constrained calculations.

Focusing first on the unconstrained calculations we see that $\mathrm{Mn}, \mathrm{Fe}$, and Co all display magnetically induced forces in the order of $0.2-0.3 \mathrm{eV} / \AA$ regardless of considered crystal structure. Bcc Fe and fcc Co share the first place in the unconstrained calculations with forces of $0.26 \mathrm{eV} / \AA$. Fcc Ni and hcp Gd have low values of magnetic disorder induced force, 0.05 and $0.06 \mathrm{eV} / \AA$, respectively. The moments in bcc Cr collapse to zero in these unconstrained calculations and thus there are zero induced forces in that case.

In the unconstrained calculations a few flippings of the magnetic moments occur. By constraining the direction of the magnetic moments, we can evaluate the effect of magnetic moment flipping on the average force. There is very little difference in the force values between the unconstrained and direction constrained magnetic moments as shown in Fig. 3, suggesting that a few magnetic moment flippings do not have a pronounced effect on the forces. For the fully constrained calculations we use the magnitude of the ferromagnetic moments. In this case the results differ from the other two cases, presenting, in general, a larger value for the average force, with a more pronounced effect on itinerant magnetic systems. The exception is bcc Mn, which displays a lower average force value for the fully constrained case. The lower force value is due to the magnetic moment in the disordered state being larger than in the FM case. Likewise, the large increase in force in the fcc Co case is due to the considerably larger FM magnetic moment magnitude, having a value of $1.7 \mu_{\mathrm{B}}$ while in the disordered case the average magnetic moment is $1.07 \mu_{\mathrm{B}}$. We have used constrained calculations as a means to assess the effect of magnetic moment magnitude and direction. Based on the findings for these elements we note that unless there is a dramatic collapse of the magnetic moments in the DLM or the FM state, the increased control of the magnetic moments by external constraints does not change the 


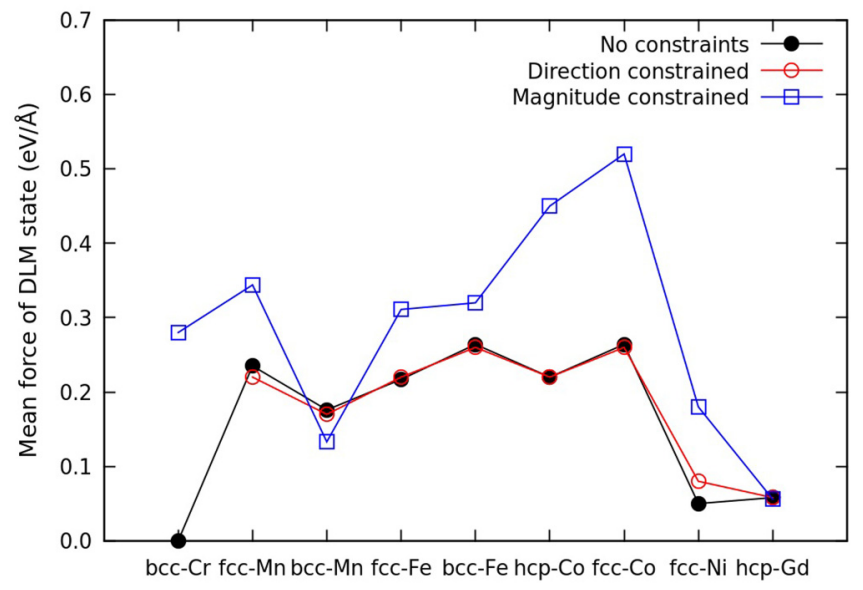

FIG. 3. Mean force for pure magnetic elements with unconstrained, direction constrained, and fully constrained magnetic moments.

relative hierarchy of magnetic disorder induced forces. Furthermore, it is artificial to demand that the moment size should be identical between different magnetic states, e.g., FM and PM, in reality. We thus performed the high-throughput search using unconstrained magnetic moments but monitored the possible appearance of moment collapses and spin flips.

\section{B. Prototype materials}

Following the elemental magnetic metals, we have tested the descriptor by probing archetypical materials for different magnetic properties of interest. FeRh is known for its magnetocaloric effect, $\mathrm{Fe}_{64} \mathrm{Ni}_{36}$ for its invar effect, and $\mathrm{BiFeO}_{3}$ for its magnetoelectric effect. In Fig. 4 we show the forces on each atom of the supercell, and the initial and final magnetic moments of each atom. In the case of FeRh, Fe atoms are subjected to larger forces than $\mathrm{Rh}$ atoms. Fe atoms possess an average magnetic moment close to $3 \mu_{\mathrm{B}}$. The magnetic moment on $\mathrm{Rh}$ atoms is small with a value close to $0.22 \mu_{\mathrm{B}}$. This compound suffered from many spin flips on the $\mathrm{Rh}$ atoms. Even if the magnetic moments are small, they prefer to be aligned in the same direction as the net magnetic moment of the cell, and we observe a similar behavior for $\mathrm{Fe}_{64} \mathrm{Ni}_{36}$. The force on $\mathrm{Fe}$ atoms was larger than $\mathrm{Ni}$, with $\mathrm{Fe}$ atoms possessing an average magnetic moment of $2.3 \mu_{\mathrm{B}}$ and $0.3 \mu_{\mathrm{B}}$ for $\mathrm{Ni}$ atoms. The ordering tendency in $\mathrm{Ni}$ atoms is stronger than in the $\mathrm{Rh}$ case for FeRh. Finally, $\mathrm{BiFeO}_{3}$ showed very large forces acting on $\mathrm{Fe}$ atoms, also large forces acting on the $\mathrm{O}$ atoms, and almost no force acting on $\mathrm{Bi}$ atoms. In this case $\mathrm{O}$ and $\mathrm{Bi}$ atoms have no magnetic moments while Fe atoms displayed a small increase from the initial $3 \mu_{\mathrm{B}}$ magnetic moment. All of these prototype compounds fulfilled the descriptor conditions, with $\mathrm{BiFeO}_{3}$ presenting the largest

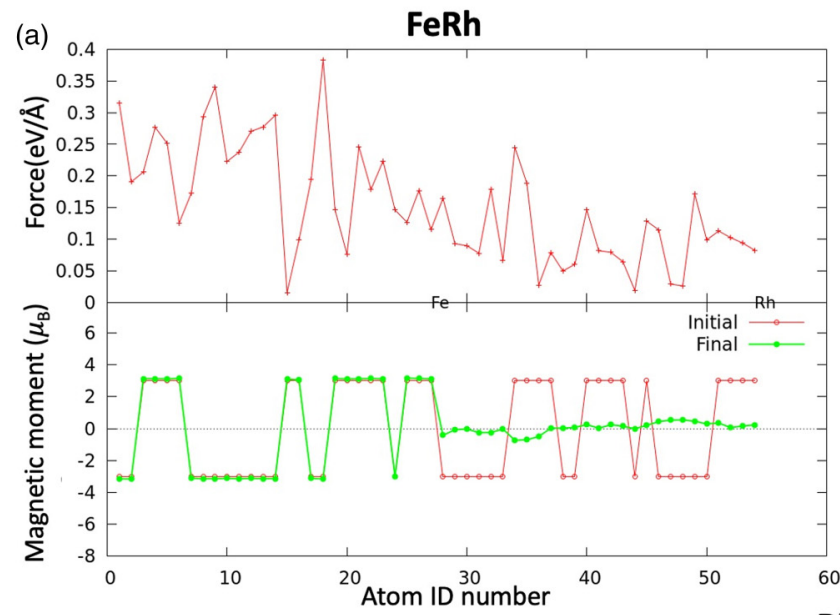

(b) 0.6

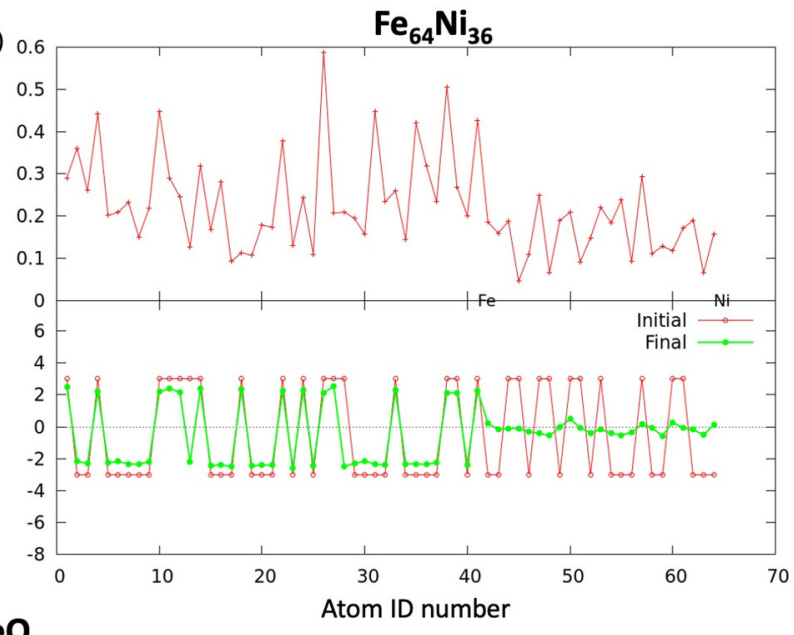

(c)

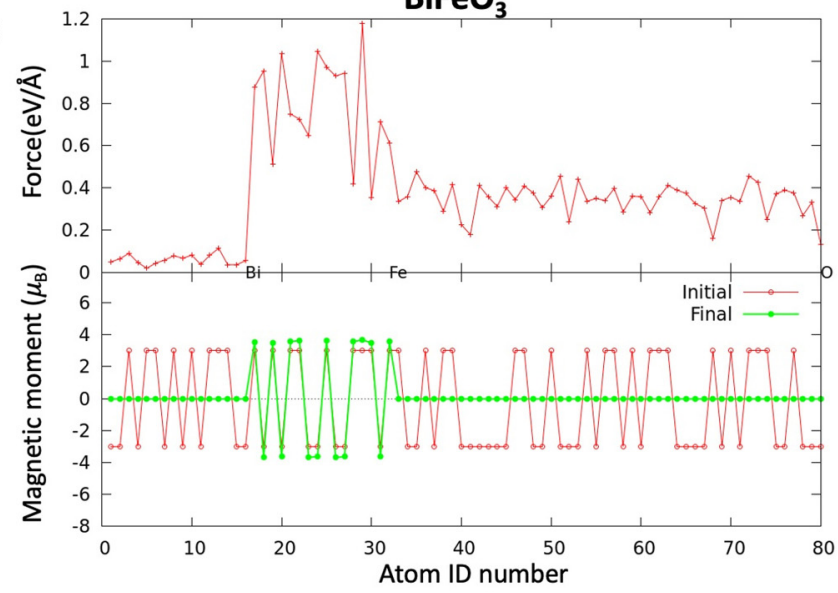

FIG. 4. Force and magnetic moments acting on each atom in (a) $\mathrm{FeRh}$, (b) $\mathrm{Fe}_{64} \mathrm{Ni}_{36}$, and (c) $\mathrm{BiFeO}_{3}$. 


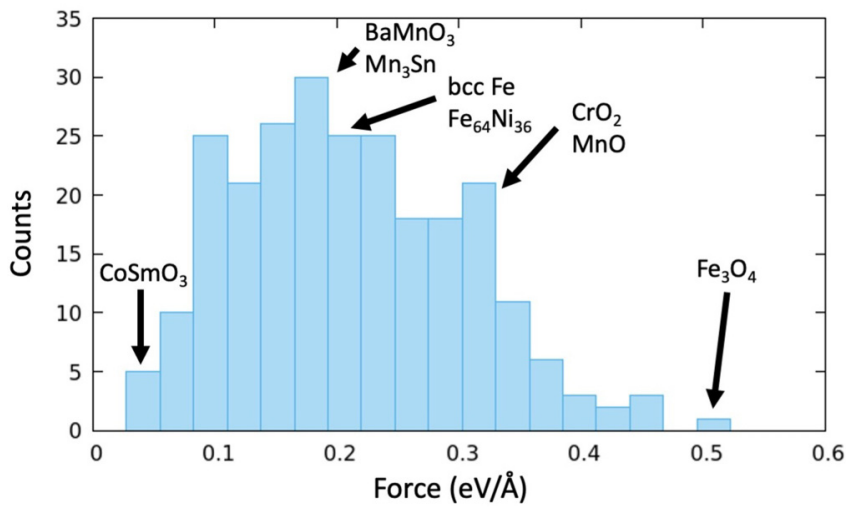

FIG. 5. Distribution of the average force acting on each atom for all the compounds in the candidate list.

average force among these model compounds, and larger than all the elements in the unconstrained results.

\section{High-throughput search}

We analyzed 1185 compounds from the COD database containing at least one $\mathrm{Fe}, \mathrm{Ni}, \mathrm{Cr}, \mathrm{Co}$, or $\mathrm{Mn}$ atomic species, with a $70 \%$ rate of successfully converged DFT calculations. We define a success as a converged DFT calculation. The main reason for nonconvergence was due to the instability created by assuming a ferromagnetic configuration for the relaxation calculations. Our scheme could be improved by incorporating the methodology of Horton et al. [28] and first search for, and then use, the obtained magnetic ground state as the starting point. From the set of successful calculations, we have 195 compounds containing Fe, 246 with Ni, 83 with $\mathrm{Cr}, 128$ with $\mathrm{Co}$, and 206 with $\mathrm{Mn}$, with 802 compounds containing only one magnetic element, and 28 with two magnetic elements. Applying our descriptor to these successfully converged calculations yielded 330 compounds of which 202 presented less than $20 \%$ spin flips.

We have ranked the candidate materials using the average force per atom given in Eq. (2), where the average is over all atomic species averaged over the two DLM configurations. In this setting a higher average force gets a higher ranking in the results list. We decided to include all atomic species, since in some cases, the highest forces in the structure are acting on the "nonmagnetic" species, e.g., $\mathrm{CrMn}_{2} \mathrm{O}_{4}$ where the strongest forces appear on the $\mathrm{O}$ atoms. We consider an element as magnetic if the magnitude of its average magnetic moment is higher than $0.05 \mu_{\mathrm{B}}$ after relaxation. In Fig. 5 we show the distribution of the average force, while in Fig. 6 we plot the distribution of the force on magnetic elements, where it can be observed that, as a general trend, Fe atoms more often experience strong magnetic disorder induced forces. In the top results list of Table I, we have included compounds that presented $20 \%$ or less spin flips. Even if our results for the elements above-and detailed constrained moment investigations of 20 additional materials-indicate that magnetic moment flipping has no strong impact on the average force, we have excluded compounds that have a large number of flips in the detailed discussion below. This is because their final magnetic state is less controlled and a material-specific

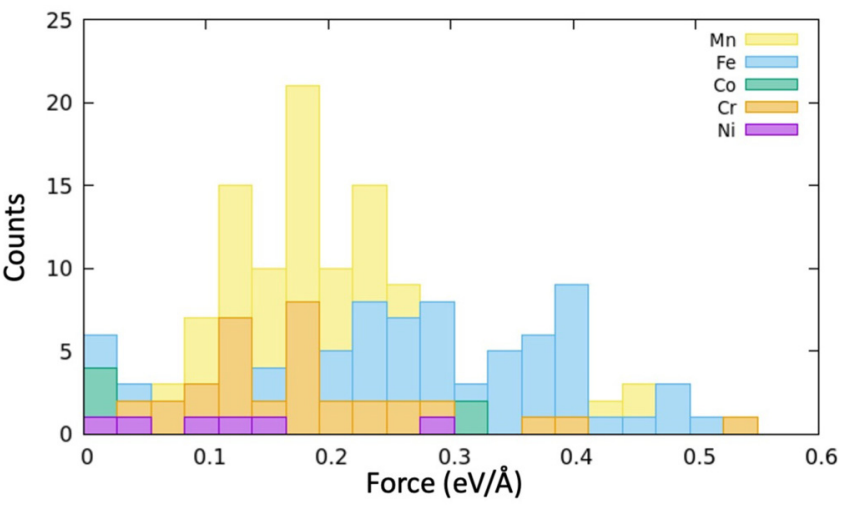

FIG. 6. Distribution of forces acting on the magnetic elements for all the compounds in the candidate list.

detailed investigation is needed for a quantitative assessment of their magnetostructural coupling in the disordered magnetic state. Nevertheless, those that display a strong average force are included with a warning remark in the full list of compounds in the Supplemental Material [48], as a service for continued future studies.

$$
\bar{F}=\frac{1}{N} \sum_{i}^{N}\left|\vec{F}_{i}\right| .
$$

We have also compared the pressure between the ferromagnetic and the DLM cases. Since we relax the ferromagnetic structure, the pressure for this phase is very close to zero.

TABLE I. Top 20 candidate materials in terms of strong magnetostructural coupling. In this table we have included compounds with $20 \%$ or less magnetic moment flips. The maximum force difference is taken as the difference in force acting on individual atoms between two DLM states

\begin{tabular}{lcc}
\hline Compound & Max. force difference $(\mathrm{eV} / \mathrm{A})$ & Average force $(\mathrm{eV} / \mathrm{A})$ \\
\hline $\mathrm{Fe}_{3} \mathrm{O}_{4}$ & 1.5156 & 0.5700 \\
$\varepsilon-\mathrm{Fe}_{2} \mathrm{O}_{3}$ & 1.4123 & 0.5270 \\
$\mathrm{Fe}_{4} \mathrm{~N}$ & 0.6483 & 0.5128 \\
$\mathrm{BaFe}_{4} \mathrm{O}_{7}$ & 1.0437 & 0.4673 \\
$\mathrm{CrMn}_{2} \mathrm{O}_{4}$ & 1.2232 & 0.4660 \\
$\mathrm{Fe}_{4} \mathrm{O}_{5}$ & 0.9972 & 0.4645 \\
$\mathrm{Fe}_{5} \mathrm{Na}_{3} \mathrm{O}_{9}$ & 1.0650 & 0.4581 \\
$\mathrm{Fe}_{7} \mathrm{O}_{9}$ & 1.2761 & 0.4307 \\
$\mathrm{CrN}$ & 1.4970 & 0.4257 \\
$\mathrm{Mn}_{3} \mathrm{NRh}_{\mathrm{NlFeO}_{3}}$ & 1.2630 & 0.4127 \\
$\mathrm{Ca}_{2} \mathrm{Fe}_{7} \mathrm{O}_{11}$ & 1.6101 & 0.4094 \\
$\mathrm{FeNaO}_{2}$ & 1.1773 & 0.4064 \\
$\mathrm{Fe}_{2} \mathrm{NaO}_{3}$ & 1.1048 & 0.4054 \\
$\mathrm{MnN}$ & 1.0035 & 0.3801 \\
$\mathrm{Fe}_{2} \mathrm{Se}_{2} \mathrm{Cs}$ & 1.1663 & 0.3765 \\
$\mathrm{Fe}_{2} \mathrm{Ge}_{2} \mathrm{Pr}$ & 1.1018 & 0.3729 \\
$\mathrm{AgFeO}_{2}$ & 0.8272 & 0.3642 \\
$\mathrm{BCe}_{2} \mathrm{Fe}$ & 0.9335 & 0.3608 \\
$\mathrm{Fe}_{2} \mathrm{~N}$ & 0.9305 & 0.3566 \\
\hline \hline
\end{tabular}



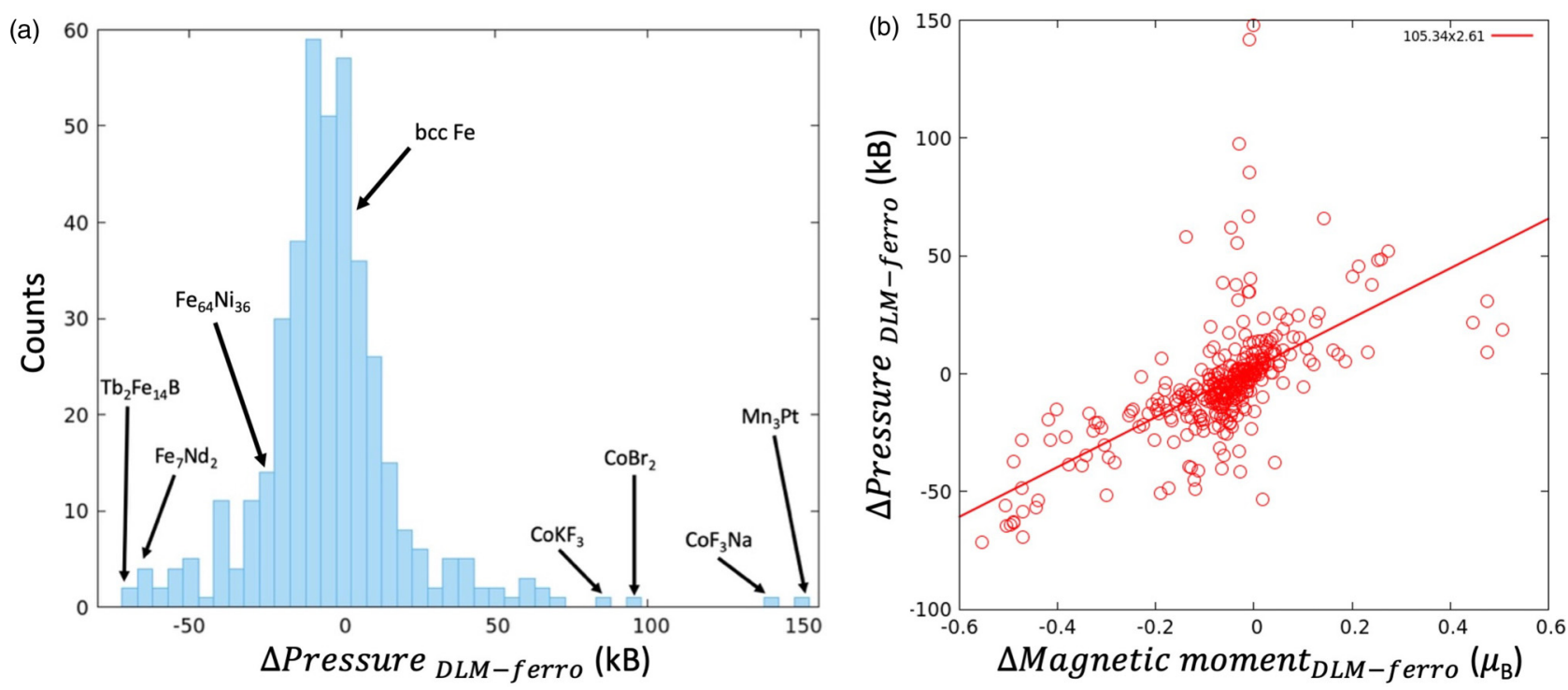

FIG. 7. (a) Distribution of the difference in pressure between the DLM and FM states for candidate compounds. (b) Pressure difference versus magnetic moment difference between DLM and FM states.

The magnetic moment magnitude changes between the ferromagnetic and the DLM states but not dramatically; therefore the pressure difference is not caused by the collapse of the magnetic moments in the DLM into a nonmagnetic state. In Fig. 7(a), we show a histogram ranking the candidate compounds according to their magnetic disorder induced pressure difference $\Delta P=P_{\mathrm{DLM}}-P_{\mathrm{FM}}$. A large pressure difference would be indicative of a driving force for a large coupling between magnetic order and/or excitations and volume change. In Fig. 7(a), we have included as a reference the values of bcc $\mathrm{Fe}$ and $\mathrm{Fe}_{64} \mathrm{Ni}_{36}$ prototype materials. $\mathrm{Fe}_{64} \mathrm{Ni}_{36}$ displays a negative pressure with respect to the ferromagnetic case for the paramagnetic high temperature limit case. This is in line with the invar behavior of $\mathrm{Fe}_{64} \mathrm{Ni}_{36}$ where some, yet not fully established, magnetic excitation away from the FM state towards the disordered state gives rise to a decrease in volume that almost exactly compensates for the normal thermal expansion due to anharmonic vibrations. Thus, the other magnetic materials observed to have a strong negative DLM pressure can be candidates for invarlike behavior. There is a relationship between the increase/decrease of the magnetic moment in the DLM state with respect to the ferromagnetic moment and the pressure of the system in the DLM state, as can be seen in in Fig. 7(b). Although most compounds follow this trend, there are some points that greatly deviate. This is a consequence of the approximation of using the ferromagnetic state as the magnetic state in the relaxation. As mentioned before, this can be improved, to a computational price, by using the actual magnetic ground state with the method proposed by Horton et al. [28]. This approximation does not affect the hierarchy of the magnetostructural coupling list, since the results for this list are obtained by examining the forces due to different DLM configurations without taking the magnetic ground state as a reference point.

The high-throughput search results yield materials that have been reported for magnetostructural coupling, and display technological relevant magnetic effects, including compounds that exhibit magnetostriction, magnetoelectricity, piezomagnetism, and superconductivity. The identification of these known compounds gives us confidence that the proposed descriptor is able to identify materials with magnetostructural coupling. In Table I we present the top 20 candidates from our high-throughput search. In this list we have only included compounds that present $20 \%$ or less spin flips of the local magnetic moments. Not surprisingly, our prototype material $\mathrm{CrN}$ is highly ranked in the list and is included among the 20 highest ranked candidate materials. Among the pure elements the highest ranking are bcc Fe and fcc Co tied at place 93. Fcc Mn, hcp Co, and fcc Fe occupy the 117, 135, and 138 positions, respectively. Bcc Mn ranks lower in the list at the 196th place, while fcc $\mathrm{Ni}$, hcp $\mathrm{Gd}$, and bcc $\mathrm{Cr}$ are at the bottom of the list. The full list is provided in the Supplemental Material [48], we also as a service to chemists present the top candidates for the oxides, nitrides and halogen compounds included in the search as an appendix at the end of the article.

In contrast to the extensively researched $\mathrm{CrN}$ and $\mathrm{Fe}_{3} \mathrm{O}_{4}$, compounds among the top candidates such as $\mathrm{CrMn}_{2} \mathrm{O}_{4}$ and $\mathrm{Ca}_{2} \mathrm{Fe}_{7} \mathrm{O}_{11}$ have less comprehensive literature, with investigations mainly devoted to characterization [49,50], opening the opportunity for further investigations of their magnetostructural coupling related properties.

\section{Top candidates review}

Ferrites are of interest for their magnetoelectric and ferroelectric behavior [51,52]. The top candidate material according to our criteria of large average force acting on the atoms is magnetite $\mathrm{Fe}_{3} \mathrm{O}_{4}$, the first material known as magnetic to mankind. The ground state is ferrimagnetic with antiparallel spin orientation on tetrahedral to octahedral sites, and has a Curie temperature of $860 \mathrm{~K}$. Magnetite undergoes a first-order metal to insulator Verwey transition at $120 \mathrm{~K}$ [53]. Proof of ferroelectric order in magnetite films has been presented [54], making magnetite a multiferroic material. $\mathrm{Fe}_{3} \mathrm{O}_{4}$ has been employed for magnetoelectric composites, incorporated as nanoparticles in a ferroelectric $\mathrm{P}(\mathrm{VDF}-\mathrm{TrFE})$ 
polymer host [55]. $\mathrm{Fe}_{3} \mathrm{O}_{4}$ has also been considered for actuators, sensors, and vibration energy harvesting systems for its magnetostrictive properties [56]. Experimentally $\mathrm{Fe}_{3} \mathrm{O}_{4}$ has displayed a magnetostriction of $23 \mathrm{ppm}$ [57], almost the same magnitude as bcc Fe (20 ppm) but with opposite sign. $\varepsilon-\mathrm{Fe}_{2} \mathrm{O}_{3}$ occupied the second position in the candidate list; it possesses an orthorhombic crystal structure, and undergoes a magnetic transition from a collinear ferrimagnetic ordering to an incommensurate magnetic state occurring at $100 \mathrm{~K}$, where there is $\sim 30 \%$ change of permittivity, suggesting the existence of magnetoelectric coupling. Gich et al. have shown that in $\varepsilon-\mathrm{Fe}_{2} \mathrm{O}_{3}$ nanoparticles the dielectric permittivity can be modified by application of a magnetic field [58]. $\varepsilon-\mathrm{Fe}_{2} \mathrm{O}_{3}$ is an attractive material since it presents advantages in terms of control of stoichiometry and stable chemical composition. $\mathrm{BaFe}_{4} \mathrm{O}_{7}$ occupies the fourth position. Two different polymorphs have been reported, hexagonal antiferromagnetic $\mathrm{BaFe}_{4} \mathrm{O}_{7}$ with a transition temperature of $945 \mathrm{~K}$ [59], and trigonal canted antiferromagnetic $\mathrm{BaFe}_{4} \mathrm{O}_{7}$ with a transition temperature of $850 \mathrm{~K}$ [51].

The corrosion and wear resistance of iron nitrides make them important for the behavior of steels, relevant for coatings and mechanical applications, but also their magnetic properties are of technological interest. Fcc structured $\mathrm{Fe}_{4} \mathrm{~N}$ has been considered for high-density recording materials, spintronics, and as a low-cost core material for transformers $[60,61]$. The average magnetic moment per iron atom in $\mathrm{Fe}_{4} \mathrm{~N}$ is close to the value of bcc alpha iron of $2.22 \mu_{\mathrm{B}}$ [62]. The magnetic structure of $\mathrm{Fe}_{4} \mathrm{~N}$ is ferromagnetic with a Curie point at $761 \mathrm{~K}$ [63]. The crystal structure of $\mathrm{Fe}_{4} \mathrm{~N}$ has been under debate; the structure reported in the COD and thus used here places the $\mathrm{N}$ atom tetrahedrally coordinated while the structure reported in other databases places $\mathrm{N}$ in an octahedron environment. DFT calculations indicate that the octahedron environment is the most stable structure. This might have an effect on the forces and change the position of $\mathrm{Fe}_{4} \mathrm{~N}$ on our result list. This illustrates the dependence on the database that is an inherent characteristic of any high-throughput study.

$\mathrm{CrN}$ has been widely used for coatings in cutting tool technologies for its hardness, but also displays strong magnetostructural coupling. As mentioned in the Introduction $\mathrm{CrN}$ undergoes a magnetic and structural transition at the critical temperature from a cubic paramagnetic to an orthorhombic antiferromagnetic phase. CrN displays an anomalous thermal conductivity [64], with an almost constant value above the magnetic critical temperature due to the coupling between spin and lattice degrees of freedom [17], and mechanical properties such as the bulk modulus depend strongly on magnetic effects $[1,2]$.

Transition-metal chalcogenides have been of research interest for their potential applications in thermoelectrics [65-67], nonlinear optics [68], and superconductivity [69,70]. Chromites have been revealed to have spontaneous electrical polarization at temperatures lower than ferrimagnetic transitions [50]. This effect makes $\mathrm{Mn}_{2} \mathrm{CrO}_{4}$ and the more famous $\mathrm{CoCr}_{2} \mathrm{O}_{4}$ attractive in spintronic applications. The transitionmetal spinels of $A B_{2} X_{4}$ general formula form a large class of materials with very rich orbital/charge/spin-ordering phenomena [71]. Recently, magnetic spinel oxides have been reported to exhibit dielectric anomaly and ferroelectric properties, both related to their magnetism [72-75].

Manganese based antiperovskites display several attractive properties such as magnetovolume effects [76-78], magnetostriction $[79,80]$, magnetocaloric effects $[81,82]$, and magnetoresistance $[83,84]$. The spin-lattice coupling displayed by $\mathrm{Mn}_{3} \mathrm{RhN}$ and $\mathrm{Mn}$ based antiperovskite nitrides are of particular interest in emerging nonvolatile magnetic random-access memory (MRAM) devices, through their piezomagnetism by probing and controlling the AFM ordering via the strain induced magnetic moment [4].

$\mathrm{Na}_{3} \mathrm{Fe}_{5} \mathrm{O}_{9}$ has monoclinic structure and is antiferromagnetic with a Néel temperature of $381 \mathrm{~K}$ [85]. $\mathrm{NaFe}_{2} \mathrm{O}_{3}$ is antiferromagnetic with a Néel temperature of $230 \mathrm{~K}$, and displays charge ordering at $250 \mathrm{~K}$. $\mathrm{NaFe}_{2} \mathrm{O}_{3}$ has been intensively studied for its potential to exhibit exotic ground states realized by the influence of spin frustration [86]. $\mathrm{NaFeO}_{2}$ crystallizes in different phases called $\alpha, \beta$, and $\gamma$. The $\alpha$ and $\beta$ phases have been studied for their ferroelectric properties [87]. $\beta-\mathrm{NaFeO}_{2}$ is a ferroelectric and weak ferromagnetic compound with ordering temperatures above room temperature. This compound has the potential to become a top candidate for applications in spintronics [87], and memory elements where electrical writing and magnetic reading would present a good balance with respect to energy consumption. $\alpha-\mathrm{NaFeO}_{2}$ has a rocksalt crystal structure with space group $R \overline{3} m$, with two magnetic phase transitions at 10.5 and $5 \mathrm{~K}$ in zero magnetic field.

\section{CONCLUSIONS}

In this work, we have proposed an efficient screening procedure to identify potential materials with strong spin-lattice coupling using a descriptor based on the force acting on each atom caused by different local magnetic environments in a disordered magnetic supercell. The method focuses on the underlying atomistic and electronic structure origin in common for several magnetostructural coupling phenomena. The proposed method is not computationally demanding, allowing us to probe a large number of compounds. We show that it successfully identifies materials reported for their magnetostructural coupling related phenomena. We provide a list of candidates displaying very strong coupling, among which we find compounds that are already known to display magnetostriction, magnetovolume, magnetocaloric, and magnetoelectric phenomena, while others have not yet been considered for such applications. The top candidate according to our descriptor is $\mathrm{Fe}_{3} \mathrm{O}_{4}$. Among the top candidates we found lesser investigated compounds such as $\mathrm{CrMn}_{2} \mathrm{O}_{4}$ and $\mathrm{Ca}_{2} \mathrm{Fe}_{7} \mathrm{O}_{11}$. This work is also a starting point in establishing a materials scale for ranking the strength of their atomistic spinlattice coupling, independent of any particular macroscopic manifestation. As a continuation of this work, we suggest that the highest ranked candidates are considered for applications where the coupling of spin and lattice degrees of freedom is the key or has been suggested to be beneficial and subjected for in-depth theoretical and experimental investigations.

\section{ACKNOWLEDGMENTS}

B.A. acknowledges financial support from the Swedish Government Strategic Research Area in Materials Science on 
Functional Materials at Linköping University, Faculty Grant No. SFOMatLiU 2009 00971, as well as support from the Swedish Foundation for Strategic Research through the Future Research Leaders 6 program, Grant No. FFL 15-0290, from the Swedish Research Council (VR) through Grant No. 2019-05403, and from the Knut and Alice Wallenberg Foundation (Wallenberg Scholar Grant No. KAW-2018.0194).
R.A. acknowledges financial support from the Swedish eScience Research Centre (SeRC) and the Swedish Research Council (VR) Projects No. 2016-04810 and No. 2020-05402. The computations were enabled by resources provided by the Swedish National Infrastructure for Computing (SNIC) at NSC partially funded by the Swedish Research Council through Grant Agreement No. 2018-05973.

\section{APPENDIX: TOP CANDIDATES BY TYPE}

Max $\Delta$ force: maximum force difference between different DLM configurations.

Avg. $\Delta$ force: average of the force difference between different DLM configurations Avg. force: Average force acting on each atom.

Spin flip \%: percentage of magnetic atoms that have changed sign from their initial setting. Units in $(\mathrm{eV} / \AA)$

\begin{tabular}{|c|c|c|c|c|}
\hline \multicolumn{5}{|l|}{ Oxides } \\
\hline Compound & $\operatorname{Max} \Delta$ force. & Avg. $\Delta$ force. & Avg. force. & Spin flip \% \\
\hline $\mathrm{Fe}_{3} \mathrm{O}_{4}$ & 1.5156 & 0.6936 & 0.5700 & $8 \%$ \\
\hline $\mathrm{Fe}_{2} \mathrm{O}_{3}$ & 1.4123 & 0.6477 & 0.5270 & $19 \%$ \\
\hline $\mathrm{BaFe}_{4} \mathrm{O}_{7}$ & 1.0437 & 0.4012 & 0.4673 & $19 \%$ \\
\hline $\mathrm{CrMn}_{2} \mathrm{O}_{4}$ & 1.2232 & 0.4193 & 0.4660 & $0 \%$ \\
\hline $\mathrm{Fe}_{4} \mathrm{O}_{5}$ & 0.9972 & 0.4331 & 0.4645 & $12 \%$ \\
\hline \multicolumn{5}{|l|}{ Nitrides } \\
\hline Compound & $\operatorname{Max} \Delta$ force & Avg. $\Delta$ force. & Avg. force. & Spin flip \% \\
\hline $\mathrm{Fe}_{4} \mathrm{~N}$ & 0.6483 & 0.4064 & 0.5128 & $0 \%$ \\
\hline $\mathrm{FeN}$ & 0.8733 & 0.5797 & 0.4440 & $>20 \%$ \\
\hline $\mathrm{CrN}$ & 1.4970 & 0.6460 & 0.4257 & $3 \%$ \\
\hline $\mathrm{Mn}_{3} \mathrm{NRh}$ & 1.2630 & 0.5677 & 0.4127 & $12 \%$ \\
\hline $\mathrm{MnN}$ & 1.1663 & 0.5621 & 0.3765 & $0 \%$ \\
\hline \multicolumn{5}{|l|}{ Halogens } \\
\hline Compound & $\operatorname{Max} \Delta$ force. & Avg. $\Delta$ force. & Avg. force. & Spin flip \% \\
\hline $\mathrm{ClFeO}$ & 0.8810 & 0.2402 & 0.2904 & $6 \%$ \\
\hline $\mathrm{CoF}_{3}$ & 1.8717 & 0.4938 & 0.2841 & $>20 \%$ \\
\hline $\mathrm{Cl}_{3} \mathrm{Mn}_{8} \mathrm{O}_{10}$ & 0.6370 & 0.2455 & 0.1850 & $0 \%$ \\
\hline $\mathrm{CoF}_{3} \mathrm{~K}$ & 0.8017 & 0.2101 & 0.1676 & $12 \%$ \\
\hline $\mathrm{CoF}_{3} \mathrm{Na}$ & 1.4834 & 0.2374 & 0.1597 & $6 \%$ \\
\hline \multicolumn{5}{|c|}{ Intermetallics } \\
\hline Compound & $\operatorname{Max} \Delta$ force & Avg. $\Delta$ force. & Avg. force. & Spin flip \% \\
\hline $\mathrm{Er}_{2} \mathrm{Fe}_{17}$ & 1.0150 & 0.4182 & 0.3897 & $>20 \%$ \\
\hline $\mathrm{Fe}_{2} \mathrm{Ge}_{2} \mathrm{Pr}$ & 0.8272 & 0.2356 & 0.3642 & $0 \%$ \\
\hline $\mathrm{Fe}_{17} \mathrm{Nd}_{2}$ & 0.7767 & 0.3241 & 0.3495 & $12 \%$ \\
\hline $\mathrm{Fe}_{17} \operatorname{Pr}_{2}$ & 0.9366 & 0.3364 & 0.3402 & $8 \%$ \\
\hline $\mathrm{Fe}_{3} \mathrm{Sn}$ & 0.8956 & 0.4349 & 0.3276 & $0 \%$ \\
\hline \multicolumn{5}{|c|}{ Chalcogenides } \\
\hline Compound & $\operatorname{Max} \Delta$ force. & Avg. $\Delta$ force. & Avg. force. & Spin flip \% \\
\hline $\mathrm{CsFe}_{2} \mathrm{Se}_{2}$ & 1.1018 & 0.3221 & 0.3729 & $3 \%$ \\
\hline $\mathrm{Cr}_{2} \mathrm{~S}_{3}$ & 1.4882 & 0.3358 & 0.3237 & $12 \%$ \\
\hline $\mathrm{BaFe}_{2} \mathrm{Se}_{3}$ & 0.4277 & 0.2085 & 0.3225 & $0 \%$ \\
\hline $\mathrm{Fe}_{2} \mathrm{KSe}_{2}$ & 1.2490 & 0.4189 & 0.3185 & $9 \%$ \\
\hline $\mathrm{FeS}$ & 0.8795 & 0.3006 & 0.3128 & $>20 \%$ \\
\hline \multicolumn{5}{|l|}{ Pictinides } \\
\hline Compound & $\operatorname{Max} \Delta$ force. & Avg. $\Delta$ force. & Avg. force. & Spin flip \\
\hline $\mathrm{As}_{2} \mathrm{EuFe}_{2}$ & 0.8354 & 0.3146 & 0.3370 & $>20 \%$ \\
\hline $\mathrm{BiFeO}_{3}$ & 1.0374 & 0.3306 & 0.3282 & $6 \%$ \\
\hline $\mathrm{As}_{3} \mathrm{CaFe}_{5}$ & 0.7692 & 0.3204 & 0.3266 & $10 \%$ \\
\hline $\mathrm{Mn}_{2} \mathrm{P}$ & 0.6437 & 0.2416 & 0.3230 & $>20 \%$ \\
\hline AsLiMn & 0.6638 & 0.2389 & 0.3223 & $0 \%$ \\
\hline
\end{tabular}


[1] B. Alling, T. Marten, and I. A. Abrikosov, Questionable collapse of the bulk modulus in CrN, Nat. Mater. 9, 283 (2010).

[2] F. Rivadulla, M. Bañobre-López, C. X. Quintela, A. Piñeiro, V. Pardo, D. Baldomir, M. A. López-Quintela, J. Rivas, C. A. Ramos, and H. Salva, Reduction of the bulk modulus at high pressure in CrN, Nat. Mater. 8, 947 (2009).

[3] B. L. Gyorffy, A. J. Pindor, J. Staunton, G. M. Stocks, and H. Winter, A first-principles theory of ferromagnetic phase transitions in metals, J. Phys. F: Met. Phys. 15, 1337 (1985).

[4] J. Zemen, Z. Gercsi, and K. Sandeman, Piezomagnetism as a counterpart of the magnetovolume effect in magnetically frustrated Mn-based antiperovskite nitrides, Phys. Rev. B 96, 024451 (2017).

[5] E. R. Callen and H. B. Callen, Static magnetoelastic coupling in cubic crystals, Phys. Rev. 129, 578 (1963).

[6] E. Hristoforou and A. Ktena, Magnetostriction and magnetostrictive materials for sensing applications, J. Magn. Magn. Mater. 316, 372 (2007)

[7] Y. Kim and Y. Y. Kim, A novel Terfenol-D transducer for guided-wave inspection of a rotating shaft, Sens. Actuators, A 133, 447 (2007).

[8] M. Fiebig, Revival of the magnetoelectric effect, J. Phys. D: Appl. Phys. 38, R123 (2005).

[9] J. Ma, J. Hu, Z. Li, and C. W. Nan, Recent progress in multiferroic magnetoelectric composites: From bulk to thin films, Adv. Mater. 23, 1062 (2011).

[10] A. P. Pyatakov and A. K. Zvezdin, Magnetoelectric and multiferroic media, Phys.-Usp. 55, 557 (2012).

[11] J. Ryu, S. Priya, K. Uchino, and H.-E. Kim, Magnetoelectric effect in composites of magnetostrictive and piezoelectric materials, J. Electroceram. 8, 107 (2002).

[12] N. A. Spaldin and R. Ramesh, Advances in magnetoelectric multiferroics, Nat. Mater. 18, 203 (2019).

[13] T. Varga, A. Kumar, E. Vlahos, S. Denev, M. Park, S. Hong, T. Sanehira, Y. Wang, C. Fennie, and S. Streiffer, Coexistence of Weak Ferromagnetism and Ferroelectricity in the High Pressure $\mathrm{LiNbO}_{3}$-type Phase of $\mathrm{FeTiO}_{3}$, Phys. Rev. Lett. 103, 047601 (2009).

[14] J. Liu, T. Gottschall, K. P. Skokov, J. D. Moore, and O. Gutfleisch, Giant magnetocaloric effect driven by structural transitions, Nat. Mater. 11, 620 (2012).

[15] A. Tishin, Y. Spichkin, V. Zverev, and P. Egolf, A review and new perspectives for the magnetocaloric effect: New materials and local heating and cooling inside the human body, Int. J. Refrig. 68, 177 (2016).

[16] A. Kitanovski and P. W. Egolf, Innovative ideas for future research on magnetocaloric technologies, Int. J. Refrig. 33, 449 (2010).

[17] I. Stockem, A. Bergman, A. Glensk, T. Hickel, F. Körmann, B. Grabowski, J. Neugebauer, and B. Alling, Anomalous Phonon Lifetime Shortening in Paramagnetic CrN Caused by SpinLattice Coupling: A Combined Spin and Ab Initio Molecular Dynamics Study, Phys. Rev. Lett. 121, 125902 (2018).

[18] Y. Grosu, A. Faik, I. Ortega-Fernández, and B. D’Aguanno, Natural Magnetite for thermal energy storage: Excellent thermophysical properties, reversible latent heat transition and controlled thermal conductivity, Sol. Energy Mater Sol. Cells 161, 170 (2017).

[19] L. Casillas-Trujillo, D. Gambino, L. Ventelon, and B. Alling, Screw dislocation core structure in the paramagnetic state of bcc iron from first-principles calculations, Phys. Rev. B 102, 094420 (2020).

[20] G. Ceder and K. Persson, How supercomputers will yield a golden age of materials science, Sci. Am. 309, 36 (2013).

[21] S. Curtarolo, G. L. W. Hart, M. B. Nardelli, N. Mingo, S. Sanvito, and O. Levy, The high-throughput highway to computational materials design, Nat. Mater. 12, 191 (2013).

[22] G. L. Hart, S. Curtarolo, T. B. Massalski, and O. Levy, Comprehensive Search for New Phases and Compounds in Binary Alloy Systems Based on Platinum-Group Metals, Using a Computational First-Principles Approach, Phys. Rev. X 3, 041035 (2013).

[23] K. Yang, W. Setyawan, S. Wang, M. B. Nardelli, and S. Curtarolo, A search model for topological insulators with highthroughput robustness descriptors, Nat. Mater. 11, 614 (2012).

[24] D. Goll, R. Loeffler, J. Herbst, R. Karimi, and G. Schneider, High-throughput search for new permanent magnet materials, J. Phys.: Condens. Matter Phys. 26, 064208 (2014).

[25] M. Jaime, A. Saúl, M. Salamon, V. Zapf, N. Harrison, T. Durakiewicz, J. Lashley, D. Andersson, C. Stanek, and J. Smith, Piezomagnetism and magnetoelastic memory in uranium dioxide, Nat. Commun. 8, 99 (2017).

[26] M. L. Green, I. Takeuchi, and J. R. Hattrick-Simpers, Applications of high throughput (combinatorial) methodologies to electronic, magnetic, optical, and energy-related materials, J. Appl. Phys. 113, 231101 (2013).

[27] A. G. Kusne, T. Gao, A. Mehta, L. Ke, M. C. Nguyen, K.-M Ho, V. Antropov, C.-Z. Wang, M. J. Kramer, and C. Long, On-the-fly machine-learning for high-throughput experiments: Search for rare-earth-free permanent magnets, Sci. Rep. 4, 6367 (2014).

[28] M. K. Horton, J. H. Montoya, M. Liu, and K. A. Persson, Highthroughput prediction of the ground-state collinear magnetic order of inorganic materials using density functional theory, npj Comput. Mater. 5, 64 (2019).

[29] J. D. Bocarsly, E. E. Levin, C. A. Garcia, K. Schwennicke, S. D. Wilson, and R. Seshadri, A simple computational proxy for screening magnetocaloric compounds, Chem. Mater. 29, 1613 (2017).

[30] R. Armiento, Database-driven high-throughput calculations and machine learning models for materials design, in Machine Learning Meets Quantum Physics, edited by K. T. Schütt, S. Chmiela, O. A. von Lilienfeld, A. Tkatchenko, K. Tsuda, and K.-R. Müller, Lecture Notes in Physics (Springer International Publishing, Cham, 2020), pp. 377-395, https://httk.org.

[31] F. Körmann, A. Dick, B. Grabowski, T. Hickel, and J. Neugebauer, Atomic forces at finite magnetic temperatures: Phonons in paramagnetic iron, Phys. Rev. B 85, 125104 (2012).

[32] B. Alling, Theory of the ferromagnetism in $\mathrm{Ti}_{1-x} \mathrm{Cr}_{x} \mathrm{~N}$ solid solutions, Phys. Rev. B 82, 054408 (2010).

[33] B. Alling, T. Marten, and I. Abrikosov, Effect of magnetic disorder and strong electron correlations on the thermodynamics of CrN, Phys. Rev. B 82, 184430 (2010).

[34] A. Zunger, S.-H. Wei, L. Ferreira, and J. E. Bernard, Special Quasirandom Structures, Phys. Rev. Lett. 65, 353 (1990).

[35] I. A. Abrikosov, A. V. Ponomareva, P. Steneteg, S. A. Barannikova, and B. Alling, Recent progress in simulations of the paramagnetic state of magnetic materials, Curr. Opin. Solid State Mater. Sci. 20, 85 (2016). 
[36] D. Gambino and B. Alling, Lattice relaxations in disordered Febased materials in the paramagnetic state from first principles, Phys. Rev. B 98, 064105 (2018).

[37] A. Filippetti and N. A. Hill, Magnetic Stress as a Driving Force of Structural Distortions: The Case of CrN, Phys. Rev. Lett. 85, 5166 (2000).

[38] J. D. Bocarsly, E. E. Levin, S. A. Humphrey, T. Faske, W. Donner, S. D. Wilson, and R. Seshadri, Magnetostructural coupling drives magnetocaloric behavior: The case of $\mathrm{MnB}$ versus FeB, Chem. Mater. 31, 4873 (2019).

[39] J. Browne, P. Liddell, R. Street, and T. Mills, An investigation of the antiferromagnetic transition of $\mathrm{CrN}$, Phys. Status Solidi A 1, 715 (1970).

[40] L. Corliss, N. Elliott, and J. Hastings, Antiferromagnetic structure of CrN, Phys. Rev. 117, 929 (1960).

[41] P. S. Herle, M. Hegde, N. Vasathacharya, S. Philip, M. R. Rao, and T. Sripathi, Synthesis of TiN, VN, and CrN from ammonolysis of $\mathrm{TiS}_{2}, \mathrm{VS}_{2}$, and $\mathrm{Cr}_{2} \mathrm{~S}_{3}$, J. Solid State Chem. 134, 120 (1997).

[42] R. Ibberson and R. Cywinski, The magnetic and structural transitions in $\mathrm{CrN}$ and (CrMo) N, Phys. B (Amsterdam, Neth.) 180, 329 (1992).

[43] S. Gražulis, D. Chateigner, R. T. Downs, A. Yokochi, M. Quirós, L. Lutterotti, E. Manakova, J. Butkus, P. Moeck, and A. Le Bail, Crystallography Open Database-an open-access collection of crystal structures, J. Appl. Crystallogr. 42, 726 (2009).

[44] G. Kresse and J. Furthmüller, Efficiency of ab-initio total energy calculations for metals and semiconductors using a plane-wave basis set, Comput. Mater. Sci. 6, 15 (1996).

[45] G. Kresse and J. Furthmüller, Efficient iterative schemes for ab initio total-energy calculations using a plane-wave basis set, Phys. Rev. B 54, 11169 (1996).

[46] J. P. Perdew, K. Burke, and M. Ernzerhof, Generalized Gradient Approximation Made Simple, Phys. Rev. Lett. 77, 3865 (1996).

[47] P. E. Blöchl, Projector augmented-wave method, Phys. Rev. B 50, 17953 (1994).

[48] See Supplemental Material at http://link.aps.org/supplemental/ 10.1103/PhysRevMaterials.5.034417 for full results list.

[49] L. Y. Gavrilova, T. Aksenova, and V. Cherepanov, Phase equilibria and crystal structures of complex oxides in systems La- $M$-Fe-O $(M=\mathrm{Ca}$ or Sr), Russ. J. Inorg. Chem. 53, 953 (2008).

[50] Y.-C. Jhuang, K. Kuo, and G. Chern, Structural and magnetic characterizations of $\mathrm{Mn}_{2} \mathrm{CrO}_{4}$ and $\mathrm{MnCr}_{2} \mathrm{O}_{4}$ films on $\mathrm{MgO}$ (001) and $\mathrm{SrTiO}_{3}$ (001) substrates by molecular beam epitaxy, J. Appl. Phys. 109, 07D714 (2011).

[51] T. Ferreira, G. Morrison, W. M. Chance, S. Calder, M. D. Smith, and H.-C. zur Loye, $\mathrm{BaFe}_{4} \mathrm{O}_{7}$ and $\mathrm{K}_{0.22} \mathrm{Ba}_{0.89} \mathrm{Fe}_{4} \mathrm{O}_{7}$ : Canted antiferromagnetic diferrites with exceptionally high magnetic ordering temperatures, Chem. Mater. 29, 2689 (2017).

[52] G. Zhang, J. Hou, M. Zhu, G. Huang, D. Li, Y. Fang, and T. Zeng, Visible-light photovoltaic effect in high-temperature ferroelectric $\mathrm{BaFe}_{4} \mathrm{O}_{7}$, J. Mater. Chem. C 8, 16234 (2020).

[53] F. Walz, The Verwey transition-a topical review, J. Phys.: Condens. Matter 14, R285 (2002).

[54] M. Alexe, M. Ziese, D. Hesse, P. Esquinazi, K. Yamauchi, T. Fukushima, S. Picozzi, and U. Gösele, Ferroelectric switching in multiferroic magnetite $\left(\mathrm{Fe}_{3} \mathrm{O}_{4}\right)$ thin films, Adv. Mater. 21, 4452 (2009).
[55] R. Belouadah, L. Seveyrat, D. Guyomar, B. Guiffard, and F. Belhora, Magnetoelectric coupling in $\mathrm{Fe}_{3} \mathrm{O}_{4} / \mathrm{P}$ (VDF-TrFE) nanocomposites, Sens. Actuators, A 247, 298 (2016).

[56] D. Odkhuu, P. Taivansaikhan, W. S. Yun, and S. C. Hong, A first-principles study of magnetostrictions of $\mathrm{Fe}_{3} \mathrm{O}_{4}$ and $\mathrm{CoFe}_{2} \mathrm{O}_{4}$, J. Appl. Phys. 115, 17A916 (2014).

[57] R. M. Bozorth, E. F. Tilden, and A. J. Williams, Anisotropy and magnetostriction of some ferrites, Phys. Rev. 99, 1788 (1955).

[58] M. Gich, C. Frontera, A. Roig, J. Fontcuberta, E. Molins, N. Bellido, C. Simon, and C. Fleta, Magnetoelectric coupling in $\varepsilon$ - $\mathrm{Fe}_{2} \mathrm{O}_{3}$ nanoparticles, Nanotechnology 17, 687 (2006).

[59] S. Okamoto, H. Sekizawa, and S. I. Okamoto, Hydrothermal synthesis, structure and magnetic properties of barium diferrite, J. Phys. Chem. Solids 36, 591 (1975).

[60] T. Monson, B. Zheng, Y. Zhou, E. J. Lavernia, C. J. Pearce, and S. Atcitty, $\gamma^{\prime}-\mathrm{Fe}_{4} \mathrm{~N}$, a New Soft Magnetic Material for Inductors and Motors, Report No. SAND2016-8042C (Sandia National Lab, Albuquerque, NM, 2016).

[61] T. E. Stevens, C. J. Pearce, M. A. Rodriguez, S. Dickens, B. B. McKenzie, S. Atcitty, and T. Monson, Synthesis of $\gamma^{\prime}-\mathrm{Fe}_{4} \mathrm{~N} a$ New Soft Magnetic Material for Inductors and Transformers, Report No. SAND2018-5099C (Sandia National Lab, Albuquerque, NM, 2018).

[62] S. Chen, S. Jin, T. Tiefel, Y. Hsieh, E. Gyorgy, and D. Johnson, Jr., Magnetic properties and microstructure of $\mathrm{Fe}_{4} \mathrm{~N}$ and $(\mathrm{Fe}, \mathrm{Ni})_{4} \mathrm{~N}$, J. Appl. Phys. 70, 6247 (1991).

[63] B. Frazer, Magnetic structure of $\mathrm{Fe}_{4} \mathrm{~N}$, Phys. Rev. 112, 751 (1958).

[64] O. Jankovský, D. Sedmidubský, Š. Huber, P. Šimek, and Z. Sofer, Synthesis, magnetic and transport properties of oxygenfree CrN ceramics, J. Eur. Ceram. Soc. 34, 4131 (2014).

[65] M. G. Kanatzidis, Nanostructured thermoelectrics: The new paradigm? Chem. Mater. 22, 648 (2009).

[66] A. Maignan, E. Guilmeau, F. Gascoin, Y. Bréard, and V. Hardy, Revisiting some chalcogenides for thermoelectricity, Sci. Technol. Adv. Mater. 13, 053003 (2012).

[67] G. Tan, L.-D. Zhao, and M. G. Kanatzidis, Rationally designing high-performance bulk thermoelectric materials, Chem. Rev. 116, 12123 (2016).

[68] I. Chung and M. G. Kanatzidis, Metal chalcogenides: A rich source of nonlinear optical materials, Chem. Mater. 26, 849 (2013).

[69] D. C. Johnston, The puzzle of high temperature superconductivity in layered iron pnictides and chalcogenides, Adv. Phys. 59, 803 (2010).

[70] H. K. Vivanco and E. E. Rodriguez, The intercalation chemistry of layered iron chalcogenide superconductors, J. Solid State Chem. 242, 3 (2016).

[71] K. Singh, A. Maignan, C. Simon, and C. Martin, $\mathrm{FeCr}_{2} \mathrm{O}_{4}$ and $\mathrm{CoCr}_{2} \mathrm{O}_{4}$ spinels: Multiferroicity in the collinear magnetic state? Appl. Phys. Lett. 99, 172903 (2011).

[72] Y. J. Choi, J. Okamoto, D. Huang, K. Chao, H. Lin, C. Chen, M. Van Veenendaal, T. Kaplan, and S.-W. Cheong, Thermally or Magnetically Induced Polarization Reversal in the Multiferroic $\mathrm{CoCr}_{2} \mathrm{O}_{4}$, Phys. Rev. Lett. 102, 067601 (2009).

[73] G. Lawes, B. Melot, K. Page, C. Ederer, M. Hayward, T. Proffen, and R. Seshadri, Dielectric anomalies and spiral magnetic order in $\mathrm{CoCr}_{2} \mathrm{O}_{4}$, Phys. Rev. B 74, 024413 (2006).

[74] N. Mufti, A. Nugroho, G. Blake, and T. Palstra, Magnetodielectric coupling in frustrated spin systems: The spinels $M \mathrm{Cr}_{2} \mathrm{O}_{4}$ 
( $M=$ Mn, Co and Ni), J. Phys.: Condens. Matter 22, 075902 (2010).

[75] Y. Yamasaki, S. Miyasaka, Y. Kaneko, J.-P. He, T. Arima, and Y. Tokura, Magnetic Reversal of the Ferroelectric Polarization in a Multiferroic Spinel Oxide, Phys. Rev. Lett. 96, 207204 (2006).

[76] D. Fruchart and E. F. Bertaut, Magnetic studies of the metallic perovskite-type compounds of manganese, J. Phys. Soc. Jpn. 44, 781 (1978).

[77] T. Kaneko, T. Kanomata, and K. Shirakawa, Pressure effect on the magnetic transition temperatures in the intermetallic compounds $\mathrm{Mn}_{3} M \mathrm{C}(M=\mathrm{Ga}, \mathrm{Zn}$ and $\mathrm{Sn})$, J. Phys. Soc. Jpn. 56, 4047 (1987).

[78] K. Takenaka, M. Ichigo, T. Hamada, A. Ozawa, T. Shibayama, T. Inagaki, and K. Asano, Magnetovolume effects in manganese nitrides with antiperovskite structure, Sci. Technol. Adv. Mater. 15, 015009 (2014).

[79] K. Asano, K. Koyama, and K. Takenaka, Magnetostriction in $\mathrm{Mn}_{3} \mathrm{CuN}$, Appl. Phys. Lett. 92, 161909 (2008).

[80] K. Takenaka, T. Shibayama, D. Kasugai, and T. Shimizu, Giant field-induced distortion in $\mathrm{Mn}_{3} \mathrm{SbN}$ at room temperature, Jpn. J. Appl. Phys. 51, 043001 (2012).

[81] T. Tohei, H. Wada, and T. Kanomata, Negative magnetocaloric effect at the antiferromagnetic to ferromagnetic transition of $\mathrm{Mn}_{3} \mathrm{GaC}$, J. Appl. Phys. 94, 1800 (2003).
[82] B. Wang, P. Tong, Y. Sun, X. Luo, X. Zhu, G. Li, X. Zhu, S. Zhang, Z. Yang, and W. Song, Large magnetic entropy change near room temperature in antiperovskite $\mathrm{SnCMn}_{3}, \mathrm{EPL} \mathbf{8 5}$, 47004 (2009).

[83] K. Kamishima, T. Goto, H. Nakagawa, N. Miura, M. Ohashi, N. Mori, T. Sasaki, and T. Kanomata, Giant magnetoresistance in the intermetallic compound $\mathrm{Mn}_{3} \mathrm{GaC}$, Phys. Rev. B 63, 024426 (2000).

[84] B. S. Wang, P. Tong, Y. P. Sun, L. J. Li, W. Tang, W. J. Lu, X. B. Zhu, Z. R. Yang, and W. H. Song, Enhanced giant magnetoresistance in Ni-doped antipervoskite compounds $\mathrm{GaCMn}_{3-x} \mathrm{Ni}_{x}$ $(x=0.05,0.10)$, Appl. Phys. Lett. 95, 222509 (2009).

[85] C. Romers, C. Rooymans, and R. De Graaf, The preparation, crystal structure and magnetic properties of $\mathrm{Na}_{3} \mathrm{Fe}_{5} \mathrm{O}_{9}$, Acta Crystallogr. 22, 766 (1967).

[86] S. Kobayashi, H. Ueda, C. Michioka, K. Yoshimura, S. Nakamura, T. Katsufuji, and H. Sawa, Anomalous double-stripe charge ordering in $\beta-\mathrm{NaFe}_{2} \mathrm{O}_{3}$ with double triangular layers consisting of almost perfect regular $\mathrm{Fe}_{4}$ tetrahedra, Phys. Rev. Mater. 2, 054402 (2018).

[87] M. Viret, D. Rubi, D. Colson, D. Lebeugle, A. Forget, P. Bonville, G. Dhalenne, R. Saint-Martin, G. Andre, and F. Ott, $\beta$ - $\mathrm{NaFeO}_{2}$, a new room-temperature multiferroic material, Mater. Res. Bull. 47, 2294 (2012). 